\title{
SOLIDARIDAD Y TRANSPARENCIA DIGITAL. WEBS Y REDES SOCIALES DE LAS ONGS ESPAÑOLAS DE ACCIÓN SOCIAL
}

\author{
Solidarity and digital transparency. Websites and \\ social networks of the Spanish social action NGOs
}

\section{Xosé Baamonde-Silva, Silvia García-Mirón y Xabier Martínez-Rolán}

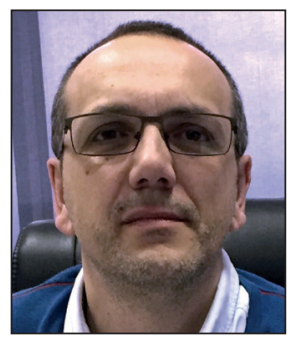

Xosé Baamonde-Silva es doctor en Ciencias de la Información por la Universidad de Santiago de Compostela. Autor de varios libros y artículos en revistas del sector comunicativo, su labor profesional estuvo ligada a la gestión de la comunicación y las relaciones públicas en el ámbito empresarial e institucional. Desde 2008 se dedica a la docencia y actualmente es profesor contratado doctor en el Departamento de Comunicación Audiovisual y Publicidad de la Universidad de Vigo y ejerce de decano de la Facultad de Ciencias Sociales y de la Comunicación de la misma universidad. http://orcid.org/0000-0003-4730-3298

xbaamonde@uvigo.es

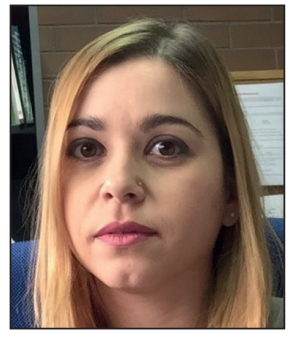

Silvia García-Mirón es doctora en Comunicación Audiovisual y Publicidad por la Universidad de Vigo y profesora ayudante doctora de la Facultad de Ciencias Sociales y de la Comunicación de la misma universidad. Ha publicado artículos y presentado ponencias sobre el estudio del medio televisivo (programación, identidad, creación de sinergias, etc.) y el análisis de estrategias publicitarias. Ha investigado acerca de la adaptación de estudios de comunicación al Espacio Europeo de Educación Superior. Es secretaria de la Facultad de Ciencias Sociales y de la Comunicación de la Universidad de Vigo.

http://orcid.org/0000-0001-8951-6051

silviamiron@uvigo.es

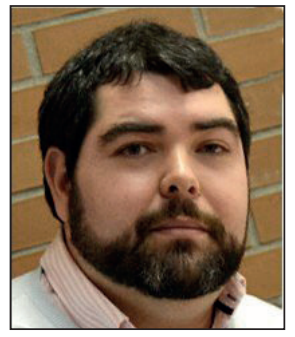

Xabier Martínez-Rolán es doctor en Comunicación por la Universidad de Vigo y profesor asociado en la Facultad de Ciencias Sociales y de la Comunicación en dicha universidad. Como gestor de comunidades online en el ámbito laboral, sus líneas de investigación se centran en el estudio de comunidades virtuales, uso y apropiación de webs, redes sociales y nuevos formatos publicitarios, y fórmulas comunicativas en nuevos medios.

http://orcid.org/0000-0002-7631-2292

xabier.rolan@uvigo.es

Universidad de Vigo

Facultad de Ciencias Sociales y de la Comunicación Campus A Xunqueira, s/n. 36005 Pontevedra, España

\section{Resumen}

La crisis económica ha supuesto una drástica disminución de las ayudas de las Administraciones a las ONGs, que además han perdido parte de la confianza social de la que gozaban. Pero hay una vía para lograr una mayor base social que contribuya a dotar de más ingresos y legitimidad a estas organizaciones, y esta vía reside en la transparencia y la rendición de cuentas. Esta investigación tiene como objeto comprobar si el denominado Tercer Sector de Acción Social (TSAS) está fomentando la participación de los nuevos ciudadanos digitales, por medio de una información pública basada en el concepto anglosajón de accountability y en la cultura de la transparencia. Para ello se ha realizado un análisis de contenido sobre las webs y redes sociales que utilizan estas entidades, para comprobar si aprovechan las oportunidades de rendir cuentas de sus actividades y fomentar una mayor participación e implicación de los ciudadanos en la acción solidaria.

\section{Palabras clave}

Transparencia; Rendición de cuentas; ONG; Tercer sector de acción social; Comunicación digital; Sitios web; Redes sociales. 


\begin{abstract}
The economic crisis has led to a drastic reduction in the funding granted by the public administrations to NGOs, which have also lost part of the social trust that they have enjoyed for decades. But there is another way to contribute more funding and legitimacy to these organizations, and this way is based on transparency and accountability. This research aims to determine if the Third Sector of Social Action (TSAS) is encouraging the participation of new digital citizens through public information based on the accountability and the transparency culture. For this purpose, we performed a content analysis on the social networks used by these entities in order to determine if they had taken advantage of the opportunities to be accountable for their activities, which can result in greater citizen participation and involvement in solidarity action.
\end{abstract}

\title{
Keywords
}

Transparency; Accountability; NGOs; Third sector of social action; Digital communication; Web sites; Social networks.

Baamonde-Silva, Xosé; García-Mirón, Silvia; Martínez-Rolán, Xabier (2017). “Solidaridad y transparencia digital. Webs y redes sociales de las ONGs españolas de acción social". El profesional de la información, v. 26, n. 3, pp. 438-446.

https://doi.org/10.3145/epi.2017.may.10

\section{Introducción}

Las organizaciones sociales se encuentran en un nuevo escenario caracterizado por dos elementos:

- el impacto de la crisis económica ha supuesto un menor volumen de ingresos;

- las malas prácticas de algunas entidades han repercutido en una pérdida de credibilidad de todo el sector.

Por ello el empleo de una comunicación digital basada en la transparencia y la rendición de cuentas puede ser una vía para incrementar los ingresos privados y recuperar la confianza social.

Hacia este aspecto se encamina la Ley de transparencia, acceso a la información pública y buen gobierno de 9 de diciembre de 2013 (España, 2013), que pretende ampliar y reforzar la transparencia de la actividad pública y, además de a las administraciones públicas, afecta a otras entidades y organismos, entre los que se podrían incluir las ONGs.

En el sector de las entidades sociales la transparencia resulta un factor clave, indispensable por coherencia con sus finalidades, ya que se conjugan los siguientes elementos (Molina-Rodríguez-Navas; Corcoy-Rius; Simelio-Solà, 2015, p. 212):

- financiación pública;

- donaciones privadas;

- trabajo voluntario;

- exigencia de alcanzar los resultados mediante procesos socialmente valorados, etc.

El tercer sector es un agente social y económico de gran relevancia en la actualidad. La etapa de crecimiento económico registrado hasta 2008 y la importancia dada al estado del bienestar provocaron un desarrollo vertiginoso del mismo. El volumen de inversión pública permitía un constante incremento de programas de intervención y la diversificación de los mismos, junto a la creación de un gran número de entidades grandes y pequeñas especializadas en sectores concretos.

Dentro de este macro-sector, esta investigación se centra en el tercer sector de acción social (TSAS), formado por entida- des privadas de carácter voluntario y sin ánimo de lucro que funcionan de forma autónoma y solidaria tratando de impulsar el reconocimiento y el ejercicio de los derechos sociales, de lograr la cohesión y la inclusión social en todas sus dimensiones y de evitar que determinados colectivos sociales queden excluidos de unos niveles suficientes de bienestar (Plataforma de ONG de Acción Social, 2013, p. 12). El campo de la acción social se distingue, por contraposición, al del desarrollo, que incluye a las organizaciones que trabajan en cooperación internacional.

La misión del TSAS es contribuir al desarrollo social y a la vertebración de la sociedad civil, promocionando la participación plena, la defensa de la libertad y la igualdad, la inclusión y la cohesión social de todas las personas y grupos que la integran (Navajo-Gómez, 2009, p. 106).

La eclosión del tercer sector se registró en España en la década de 1990 y en los primeros años del nuevo milenio. Durante ese período, las entidades de este sector disfrutaron de un alto grado de legitimidad y credibilidad. Sin embargo, su reputación ha ido empeorando y las ONGs no han sabido generar una cultura de la solidaridad que durante el azote de la crisis económica ha agravado una situación en la que no encuentran argumentos para que los ciudadanos sean solidarios y permanezcan junto a ellas y del lado de las causas que defienden (Burgui-Jurío, 2010, p. 179).

El impacto de la crisis económica y la correlacionada desidealización de las organizaciones no lucrativas sitúan a las ONGs de acción social ante un reto decisivo en su historia. Necesitan recuperar un alto nivel de confianza social que les permita tener una base social comprometida, y unos financiadores que les apoyen y les proporcionen recursos económicos para llevar a cabo su labor. Para lograr, mantener e incrementar esa confianza social, las organizaciones han de asumir la transparencia y la rendición de cuentas como un requisito indispensable.

\section{Estado de la cuestión}

La búsqueda de soluciones para superar la disminución de ingresos procedentes de las administraciones públicas, fruto de la crisis económica, y generar una mayor confianza 
social, conducen a las organizaciones del tercer sector a la adopción de herramientas para mejorar la información que trasladan a sus públicos.

La accountability es una decisión voluntaria de la entidad, un compromiso ético y de responsabilidad de sus actuaciones, que va más allá de la rendición obligatoria en relación con las cuentas económicas (Herranz-de-la-Casa; Cabezuelo-Lorenzo, 2009, p. 182). El concepto rendición de cuentas hace referencia a la explicación de aspectos organizativos tales como la razón de ser y los valores de la entidad, las actividades, su impacto, la financiación, el equipo, las políticas de gestión y desarrollo de personas, la visión que tiene de la sociedad, las políticas de comunicación, etc. En definitiva, es ofrecer una visión global de la organización.

Transparencia significa mostrar abiertamente el engranaje de una organización: cómo funciona, cómo se lleva a cabo su trabajo, cuáles son los procesos internos, sus decisiones y los resultados obtenidos

La transparencia se entiende como el grado de información y la actitud con que se afronta la rendición de cuentas (Balas-Lara, 2011; Gálvez-Rodríguez; Caba-Pérez; López-Godoy, 2016; Martínez-Osés et al., 2011; García-López, 2012; Herranz-de-la-Casa; Cabezuelo-Lorenzo, 2009). La transparencia debe ser coherente con la entidad y su misión. Más allá de las cuentas claras, transparencia significa mostrar abiertamente el engranaje de una organización. Es decir, cómo funciona, cómo se lleva a cabo su trabajo, cuáles son los procesos internos, sus decisiones o los resultados obtenidos. La ONG debe actuar de manera responsable social, económica y ambientalmente.

Las cuestiones referidas a la rendición de cuentas y la transparencia son entendidas con demasiada frecuencia en su versión más limitada, que consiste en atender los requerimientos de justificación financiera impuestos por los financiadores, obviando la responsabilidad de cara a los propios beneficiarios y que se informe sobre resultados e impactos en los procesos de desarrollo (Martínez-Osés et al., 2011, p. 20).

La importancia de ser transparente para las ONGs se basa en que (Balas-Lara, 2011, p. 185):

- es un valor intrínseco a su forma de actuar;

- permite tener una base social grande e implicada;

- obliga a medir los resultados de sus actuaciones y proyectos;

- facilita la comparación con otras organizaciones similares; - obliga a vigilar el cumplimiento de su misión.

Más que por imperativo legal o por interés particular para captar fondos, la transparencia y la rendición de cuentas por parte de las ONGs suponen el derecho a recibir información y la obligación de divulgar todos los datos pertinentes, con el objetivo de lograr una mayor participación e implicación social. Para ello, siguiendo a Marí-Sáez (2016, p. 155), las organizaciones solidarias impulsan dos ejes prioritarios:

"la dinamización de procesos sociales de cambio y el fomento de la participación ciudadana".

Los años de flaqueza de las ONGs han suscitado un debate crítico y multidireccional que apuesta por establecer espacios de vinculación pública entre las organizaciones y sus públicos. La Web y las redes sociales pueden ser ese lugar de encuentro para facilitar una conversación constante que redunde en una mejor acción solidaria y un mayor compromiso de los ciudadanos.

Esta cuestión ha atraído la mirada tanto de los profesionales como de la Academia, que han analizado la relación entre transparencia y participación en los últimos años (Barranquero-Carretero, 2014; Burgui-Jurío, 2010; Darnton; Kirk, 2011; García-López, 2012; Martínez-Osés et al., 2011; Narberhaus, 2011; Santolino-Prieto, 2010).

Es preciso destacar, aunque no sean muy numerosos, los estudios que relacionan las TIC, las ONGs y la participación ciudadana (Arroyo-Almaraz; Baladrón-Pazos; Martín-Nieto, 2013; Baamonde-Silva; Martínez-Rolán; Míguez-González, 2016; Barranquero-Carretero, 2014; Herranz-de-la-Casa; Cabezuelo-Lorenzo, 2009; Soria-Ibáñez, 2011).

El entorno digital parece favorecer la aparición de nuevas formas de transparencia, autorregulación y supervisión de la calidad informativa, en las que la participación de los usuarios juega un papel decisivo (Mauri-Ríos; Ramón-Vegas, 2015, p. 380).

Por ello, se ha llevado a cabo un análisis de las webs y las redes sociales de las organizaciones pertenecientes a la Plataforma de ONG de Acción Social, para tratar de comprobar si constituyen una herramienta al servicio de la transparencia y la rendición de cuentas.

\section{Materiales y métodos}

Con la finalidad de abordar el estado de la transparencia de las ONGs de acción social se realizó un estudio exploratorio de sus sitios web, empleando como método de investigación el análisis de contenido. Se elaboró una ficha de análisis propia que permitió evaluar la transparencia desde múltiples puntos de atención.

Para llevar a cabo el diseño metodológico, y más específicamente el cuestionario con el que realizar el trabajo de campo, se sentaron los cimientos en la Ley de transparencia (España, 2013), en la que se recoge que:

"establece las obligaciones de buen gobierno que deben cumplir los responsables públicos, así como las consecuencias jurídicas derivadas de su incumplimiento -lo que se convierte en una exigencia de responsabilidad para todos los que desarrollan actividades de relevancia pública",

y en esa relevancia pública incluimos la actuación y decisiones de las ONGs.

Partiendo de ahí, se tomaron como base diversos estudios centrados en transparencia, advirtiendo aquellos que más encajaban con la investigación que pretendíamos realizar: 
Mapa Infoparticipa, calidad y transparencia de la comunicación pública (Moreno-Sardà et al., 2013); TransparEnt, calidad y transparencia de la información de las entidades sin ánimo de lucro (Molina-Rodríguez-Navas; Lavado-Campàs, 2015), desarrollados por el Laboratorio de Periodismo y Comunicación para la Ciudadanía Plural de la Universitat Autònoma de Barcelona.

También se tomó como referencia el análisis de la transparencia en ayuntamientos gallegos y lusos propuesto por Martínez-Rolán, Piñeiro-Otero y Baamonde-Silva (2016).

Tras el estudio de los diversos items que abordaban en cada bloque de contenido, se efectuó una adaptación propia, siempre desde un punto de vista comunicativo y no meramente de aplicación administrativa, teniendo en consideración la realidad objeto de estudio: las ONGs de acción social.

La primera parte de la ficha de análisis consta de 28 preguntas agrupadas en cinco apartados (ver tabla 1).

- Organización interna: relativa al funcionamiento interno de las ONGs: estatutos, órganos de gobierno, responsables...

- Rendición de cuentas: información sobre los fines y actua- ciones de la entidad, así como la información económica y vías de contacto con la ONG.

- Socios/donantes/colaboradores: información específica para los mismos, área privada y posibilidad de colaboración o adhesión a la organización. Este apartado engloba a aquellos públicos vinculados con la organización sin cuya aportación ésta no puede cumplir su misión y, por tanto, merecerían un tratamiento específico. Abarca a socios, donantes, voluntarios o todo tipo de colaboradores que contribuyen a que la entidad alcance sus fines solidarios.

- Comunicación: con el análisis exhaustivo de los diferentes métodos, formatos y medios de comunicación externa

- Ética y autorregulación: donde se recogen elementos deontológicos como un código de conducta, un sello de garantía o la especificidad de los criterios de colaboración con empresas.

La importancia de las plataformas de gestión de redes sociales en la transparencia queda patente en los estudios de Karkin (2013), fuente de inspiración para la parte correspondiente a medios sociales en la ficha de investigación. Para ello, en una segunda parte de la ficha de análisis se ha contabilizado el número de plataformas sociales en las que cada entidad tiene presencia de forma activa.

Tabla 1. Ficha de análisis empleada

\begin{tabular}{|c|c|c|}
\hline Categoría & Atributo a analizar & $\begin{array}{l}\text { Puntuación por apartado } \\
\text { (un punto por pregunta) }\end{array}$ \\
\hline \multirow{3}{*}{ Organización interna } & Los estatutos son accesibles y disponibles para el público & \multirow{3}{*}{3} \\
\hline & Los órganos de gobierno están a disposición del público & \\
\hline & Los nombres de los responsables de la estructura ejecutiva son públicos & \\
\hline \multirow{10}{*}{ Rendición de cuentas } & Se explica la misión, visión y valores & \multirow{10}{*}{10} \\
\hline & Tiene un formulario para contactar con la organización & \\
\hline & Explica su historia y trayectoria & \\
\hline & Hace pública su planificación y objetivos & \\
\hline & Indica la implantación territorial de la organización (sedes) & \\
\hline & Enumera los proyectos y actividades que lleva a cabo & \\
\hline & Informa de sus zonas de actuación & \\
\hline & Puede descargarse la memoria & \\
\hline & Indica la procedencia y destino de sus recursos económicos & \\
\hline & Dispone de una auditoría externa & \\
\hline \multirow{3}{*}{ Socios / donantes / colaboradores } & Especifica el número de socios & \multirow{3}{*}{3} \\
\hline & Dispone de un formulario para hacerse socio o donar & \\
\hline & Posee un espacio virtual específico para socios, donantes o voluntarios & \\
\hline \multirow{9}{*}{ Comunicación } & Posee un espacio específico para periodistas & \multirow{9}{*}{9} \\
\hline & Utiliza un sistema de boletín electrónico & \\
\hline & Elabora y dispone de forma pública algún tipo de dossier de prensa & \\
\hline & Permite la descarga de las notas de prensa & \\
\hline & Permite descargar las imágenes o vídeos & \\
\hline & El responsable de prensa está identificado & \\
\hline & Hace uso de una o varias redes sociales & \\
\hline & Actualiza con frecuencia sus perfiles en redes sociales & \\
\hline & Dispone de foro o de blog & \\
\hline \multirow{3}{*}{ Ética y autorregulación } & Tiene accesible algún código de conducta & \multirow{3}{*}{3} \\
\hline & Dispone de criterios de colaboración con empresas & \\
\hline & Posee algún distintivo o sello de garantía de transparencia & \\
\hline
\end{tabular}




\subsection{Acotación de la muestra}

La selección de las organizaciones analizadas se basó en el listado de entidades pertenecientes a la Plataforma de ONG de Acción Social, ya que reúne a los principales actores relacionados con el objeto de estudio.

El listado actualizado de miembros de la plataforma asciende a 25 organizaciones:

- Accem

http://www.accem.es

- Asociación Española contra el Cáncer (AECC) https://www.aecc.es

- Cáritas

http://www.caritas.es

- Comisión Española de Ayuda al Refugiado (CEAR) http://cear.es

- Confederación de Centros de Desarrollo Rural (Coceder) http://www.coceder.org

- Confederación Española de Personas con Discapacidad Física y Orgánica (Cocemfe)

http://www.cocemfe.es

- Confederación Estatal de Personas Sordas (CNSE) http://www.cnse.es

- Coordinadora Estatal de Plataformas Sociales Salesianas http://www.psocialessalesianas.org

- Cruz Roja Española http://www.cruzroja.es

- Federación de Asociaciones de Scouts de España (ASDE) http://www.scout.es

- Federación de la Mujer Rural (Femur) http://www.femur.es

- Federación de Mujeres Progresistas (FMP) http://www.fmujeresprogresistas.org

- Federación Didania de Entidades Cristianas de Tiempo Libre http://www.didania.org

- Fundación Cepaim http://cepaim.org

- Fundación Esplai http://www.fundacionesplai.org

- Fundación Juan Ciudad (Orden Hospitalaria de San Juan de Dios)

http://www.juanciudad.org

- Fundación Secretariado Gitano

http://www.gitanos.org

- Liga de la Educación y la Cultura Popular http://ligaeducacion.org

- Movimiento por la Paz el Desarme y la Liberación http://www.mpdl.org

- Organización Nacional de Ciegos Españoles (ONCE) http://www.once.es
- Plena Inclusión (Antes Feaps) http://www.plenainclusion.org

- Unión de Asociaciones Familiares http://unaf.org

- Unión de Asociaciones y Entidades de Atención al Drogodependiente (UNAD)

http://www.unad.org

- Unión Democrática de Pensionistas y Jubilados de España (UDP)

https://www.mayoresudp.org

- Unión Romaní

http://www.unionromani.org

Si bien la mayor parte de los miembros se unieron en 2000, la última adhesión es de 2015 (Accem).

Se han descartado las entidades observadoras permanentes y otras entidades asociadas colaboradoras por no ser miembros de la Plataforma.

El estudio de la transparencia de las webs de estos 25 organismos se realizó durante los meses de noviembre y diciembre de 2016, de forma que los datos han sido capturados por un investigador y revisados y validados por dos investigadores más para dar fidelidad y mayor coherencia a las puntuaciones obtenidas.

\section{Análisis de resultados}

Tras la observación y análisis de los datos relativos a los 28 indicadores seleccionados, se ha advertido en primer lugar que hay 5 organizaciones que no superan el $50 \%$ de los indicadores, hecho que les impediría obtener el aprobado en cuanto a transparencia.

\section{4 de las 28 ONGs ofrecen un grado de cumplimiento mayor del $75 \%$ y una de ellas ofrece una verificación positiva en todos los items}

No obstante, se constata de forma positiva que 14 de las 28 ONGs ofrecen un grado de cumplimiento mayor del $75 \%$ e incluso una de ellas ofrece una verificación positiva en todos los items. Más del $80 \%$ de las entidades superan la mitad de los indicadores analizados.

En segundo lugar, se ha afrontado el análisis por cada uno de los grupos de indicadores y redes sociales (tabla 1). En relación con el primer grupo de indicadores analizado - organiza-

Tabla 2. Grado de cumplimiento de los indicadores por parte de las ONGs

\begin{tabular}{|l|c|}
\hline \multicolumn{1}{|c|}{ \% de indicadores cumplidos } & N. de ONGs \\
\hline Menos de 25 & 2 \\
\hline De 25 a 49 & 3 \\
\hline De 50 a 74 & 6 \\
\hline De 75 a 100 & 14 \\
\hline
\end{tabular}


Tabla 3. Indicadores sobre la organización interna de las ONGs en sus webs corporativas

\begin{tabular}{|l|c|}
\hline \multicolumn{1}{|c|}{ Indicador } & \% ONGs \\
\hline $\begin{array}{l}\text { 1. Los estatutos son accesibles y están disponibles al } \\
\text { público }\end{array}$ & 60 \\
\hline 2. Los órganos de gobierno están a disposición pública & 72 \\
\hline $\begin{array}{l}\text { 3. Los nombres de los responsables de la estructura } \\
\text { ejecutiva son públicos }\end{array}$ & 80 \\
\hline
\end{tabular}

ción interna de las ONGs- se aprecia que más de la mitad de las entidades cumplen con los tres indicadores de este grupo (tabla 3). De este modo, el 60\% sí incorporan sus estatutos en sus webs corporativas, así como los órganos de gobierno (un 72\%) y los nombres de los responsables de la estructura ejecutiva, un dato que omiten tan sólo 4 entidades.

Respecto a la rendición de cuentas (tabla 4), los resultados indican que más de la mitad de las organizaciones no explica su historia y trayectoria en su web corporativa o que sólo el $56 \%$ dispone de una auditoría externa. También resulta complejo poder consultar su planificación y objetivos.

En el lado del mayor grado de cumplimiento se constata que todas las asociaciones, con una única excepción, indican su implantación territorial (sus sedes) y sólo dos no informan de sus proyectos y actividades. Los resultados también arrojan un alto nivel de cumplimiento en lo que atañe a explicación de la misión, visión o valores (84\%), la posibilidad de consulta y descarga de la Memoria anual (84\%) o información sobre sus zonas de actuación (80\%).

\section{Las ONGs de la Plataforma Española de Acción Social dan relevancia a las redes sociales; todas hacen uso de ellas y el $96 \%$ las actualiza con frecuencia}

En la información sobre socios, donantes o colaboradores, los datos no devuelven excesiva transparencia por parte de las ONGs: sólo seis de cada diez hacen público su número de socios, donantes o voluntarios, e incluso son menos las que disponen de un espacio específico para estos públicos, esenciales para lograr los objetivos organizacionales (sólo el $56 \%$ de las entidades lo hace).

En comunicación los resultados revelan que actualmente todas las ONGs que componen la Plataforma Española de Acción Social dan relevancia a las redes sociales (tabla 6); todas hacen uso de ellas y el $96 \%$ las actualiza con frecuencia.

También destaca la incorporación de espacios específicos para periodistas en los sitios web, así como el envío de newsletters a cualquier usuario interesado. Con todo, no resulta habitual encontrar claramente identificada a la persona responsable de prensa, los dossieres de prensa o el uso de foros o blogs asociados a los contenidos corporativos de la web.

Las cuestiones relacionadas con la ética y autorregulación - códigos de conducta, criterios de colaboración con empresas o disposición de algún sello de garantía de transparencia-, ofrecen los peores resultados del análisis: en más
Tabla 4. Indicadores sobre la rendición de cuentas de las ONGs en sus webs corporativas

\begin{tabular}{|l|c|}
\hline \multicolumn{1}{|c|}{ Indicador } & \% ONGs \\
\hline 4. Explica la misión, la visión o los valores & 84 \\
\hline 5. Tiene un formulario para contactar con la organización & 76 \\
\hline 6. Explica su historia y trayectoria & 48 \\
\hline 7. Incluye su planificación y objetivos & 60 \\
\hline $\begin{array}{l}\text { 8. Indica la implantación territorial de la organización } \\
\text { (sedes) }\end{array}$ & 96 \\
\hline 9. Enumera sus proyectos y actividades & 92 \\
\hline 10. Informa de sus zonas de actuación & 80 \\
\hline 11. Permite la descarga de la memoria anual & 84 \\
\hline $\begin{array}{l}\text { 12. Indica la procedencia y destino de los recursos } \\
\text { económicos }\end{array}$ & 72 \\
\hline 13. Dispone de una auditoría externa & 56 \\
\hline
\end{tabular}

Tabla 5. Indicadores sobre los socios, donantes o colaboradores de las ONGs en sus webs corporativas

\begin{tabular}{|l|c|}
\hline \multicolumn{1}{|c|}{ Indicador } & \% ONGs \\
\hline $\begin{array}{l}\text { 14. Especifica el número de socios/donantes/colabora- } \\
\text { dores }\end{array}$ & 60 \\
\hline $\begin{array}{l}\text { 15. Dispone de un formulario para hacerse socio o donar } \\
\text { 16. Incluye un espacio específico para socios, donantes o } \\
\text { voluntarios }\end{array}$ & 68 \\
\hline
\end{tabular}

Tabla 6. Indicadores sobre la comunicación de las ONGs en sus webs corporativas

\begin{tabular}{|l|c|}
\hline \multicolumn{1}{|c|}{ Indicador } & \% ONGs \\
\hline 17. Incorpora un espacio específico para periodistas & 84 \\
\hline 18. Tiene newsletter & 84 \\
\hline 19. Ofrece dossier de prensa & 48 \\
\hline 20. Permite la descarga de las notas de prensa & 76 \\
\hline 21. Permite la descarga de imágenes o vídeos & 68 \\
\hline 22. Está identificado el responsable de prensa & 44 \\
\hline 23. Utiliza las redes sociales & 100 \\
\hline 24. Actualiza con frecuencia sus perfiles en redes sociales & 96 \\
\hline 25. Dispone de foro o de blog & 48 \\
\hline
\end{tabular}

Tabla 7. Indicadores sobre ética y autorregulación de las ONGs en sus webs corporativas

\begin{tabular}{|l|c|}
\hline \multicolumn{1}{|c|}{ Indicador } & \% ONGS \\
\hline 26. Tiene accesible algún código de conducta & 40 \\
\hline 27. Dispone de criterios de colaboración con empresas & 40 \\
\hline 28. Dispone de algún sello de garantía de transparencia & 36 \\
\hline
\end{tabular}

del $40 \%$ de la muestra analizada no aparece ningún atributo.

Finalmente, se ha prestado atención a los perfiles en redes sociales de las ONGs, concretamente en Facebook, Twitter, YouTube, Instagram, Google+, Flickr, Pinterest, LinkedIn y Vimeo. Se ha notado que la mayor parte de las entidades tienen presencia en varias plataformas -en 3 ó 4 de forma recurrente- y sólo en un caso, en 6 medios sociales. 
Tabla 8. Indicadores sobre la presencia en redes sociales de las ONGs en España

\begin{tabular}{|l|c|}
\hline \multicolumn{1}{|c|}{ Red social } & \% ONGs \\
\hline Facebook & 96 \\
\hline Twitter & 92 \\
\hline YouTube & 80 \\
\hline Instagram & 8 \\
\hline Google+ & 16 \\
\hline Flickr & 12 \\
\hline Pinterest & 4 \\
\hline Linkedln & 12 \\
\hline Vimeo & 4 \\
\hline
\end{tabular}

Se ha podido apreciar la fuerte implantación de Facebook y Twitter en las ONGs españolas de acción social (por encima del 90\%). Asimismo YouTube, que está presente en 8 de cada 10 ONGs, aparece como una de las principales plataformas empleadas. Vimeo, Pinterest e Instagram apenas tienen penetración entre las ONGs españolas.

\section{Conclusiones y discusión}

Las ONGs del tercer sector de acción social son conscientes de que la transparencia y la rendición de cuentan no son una opción sino una necesidad. Precisan dar información adecuada y pertinente para afrontar la disminución de recursos procedentes de las administraciones públicas y para lograr una mayor implicación y participación de los ciudada- nos en la actividad solidaria.

Aunque el $80 \%$ de las entidades superan el $50 \%$ de los indicadores analizados, el estudio revela el largo trecho que queda por recorrer a las ONGs de acción social.

Los datos sobre la estructura interna de la organización son positivos, al igual que la mayoría de los que se refieren a los medios para la rendición de cuentas. Cabe destacar que el $84 \%$ de las webs analizadas permiten la descarga de la memoria anual de la entidad, que puede considerarse como el instrumento básico en la gestión de la transparencia y facilitación de información pública.

Las cifras sobre la información facilitada a los socios, donantes o colaboradores y la relación con éstos, revelan cierta desidia con respecto a este público. Las webs deberían contar con espacios específicos en los que buscar una mayor vinculación con este público prioritario para cualquier ONG.

El apartado de comunicación ofrece unos altos índices de cumplimiento, propios de un sector que ya está acostumbrado a relacionarse con los periodistas para buscar una mayor visibilidad y repercusión. Por ello resulta confusa la ofuscación del contacto directo con el responsable de comunicación; una demanda mediática en aumento.

El aspecto con más margen de mejora son los sistemas de autorregulación: tan sólo el $40 \%$ dispone de un código de conducta o de unos criterios para colaborar con las empresas, y sólo una de cada tres entidades dispone de un sello de garantía de transparencia. Aunque algunas organizaciones están avaladas por la Fundación Lealtad (especializada en este campo) o por la herramienta de la Coordinadora de ONGs de Desarrollo (en funcionamiento desde 2012), sería interesante que el TSAS dispusiera de su propio instrumento para garantizar la transparencia y el buen gobierno.

El proceso de construcción de una ciudadanía participativa requiere del compromiso activo de todos, pero especialmente de las futuras generaciones, por lo que la utilización de las redes sociales se hace imprescindible. La utilización de las redes sociales por parte de las ONGs es alta: Facebook y Twitter superan el $90 \%$ y YouTube el $80 \%$, lo que avala la importancia de las imágenes para trasladar los mensajes de estas entidades, respetando la obligación moral que tienen las organizaciones de representar las distintas realidades (Alonso-Fernández, 2011, p. 445).

La aprobación de la Ley de transparencia, acceso a la información pública y buen gobierno, de 9 de diciembre de 2013, supone el inicio de una mayor exigencia a las 
administraciones públicas y a las organizaciones sociales de los datos que ponen a disposición de los ciudadanos; pero los logros no son inmediatos y los resultados obtenidos en algunos estudios sobre su cumplimiento son calificados como "una asignatura pendiente" (BeltránOrenes; Martínez-Pastor, 2016, p. 558) o "decepcionantes" (ManfrediSánchez, 2016, p. 265).

Los datos obtenidos con esta investigación no permiten ser tan taxativos a la hora de describir la transparencia en las ONGs de acción social. Con todo, este estudio se alinea con los anteriores y permite concluir que los resultados ofrecen un holgado margen de mejora para este tipo de organizaciones.

Este trabajo por otra parte, invita a continuar y ampliar el análisis de la situación de la transparencia a través de las webs corporativas de las ONGs de menores dimensiones a las que integran la Plataforma Española de Acción Social, así como a aquellas que ofrecen una actuación con carácter autonómico. Se considera el presente trabajo como el germen preliminar para una futura línea de investigación que compare los datos obtenidos con los de otros ámbitos del tercer sector, como puede ser el de la cooperación para el desarrollo e, incluso, con entidades de otras esferas económicas y sociales.

El análisis de la transparencia digital de las ONGs permite concluir que el uso de los medios sociales no garantiza nada; tan sólo adquieren sentido con la puesta en marcha de una cultura corporativa que ponga en valor la transparencia como un eje fundamental en las relaciones con la sociedad. Las ONGs del TSAS pueden liderar esta apuesta por esta vía de incrementar la información a disposición de los diferentes públicos para lograr una mayor credibilidad, legitimidad, confianza y participación social.

\section{Bibliografía}

Alonso-Fernández, Juan (2011). “Gestión y conservación de documentos fotográficos en el Tercer Sector: experiencia de la Fundación Vicente Ferrer". El profesional de la información, v. 20 n. 4, pp. 444-447.

https://doi.org/10.3145/epi.2011.jul.12

Arroyo-Almaraz, Isidoro; Baladrón-Pazos, Antonio; MartínNieto, Rebeca (2013). "La comunicación en redes sociales: percepciones y usos de las ONG españolas". Cuadernos de información, n. 32, pp. 77-88.

https://doi.org/10.7764/cdi.32.497

Baamonde-Silva, Xosé; Martínez-Rolán, Xabier; MíguezGonzález, María-Isabel (2016). "Las ONG como agentes de transformación social. Del asistencialismo a la movilización". Obets. Revista de ciencias sociales, v. 11, n. 1, pp. 53-74. https://doi.org/10.14198/OBETS2016.11.1.04
Balas-Lara, Montserrat (2011). La gestión de la comunicación en el Tercer Sector. Como mejorar la imagen de las ONG. Madrid: Esic Editorial. ISBN: 9788473568074

Barranquero-Carretero, Alejandro (2014). “Comunicación, cambio social y ONG en España. Pistas para profundizar en la cultura de la cooperación desde los nuevos movimientos comunicacionales. El caso del 15M". Commons. Revista de comunicación y ciudadanía digital, v. 3, n. 1, pp. 6-34. http://hdl.handle.net/10016/20699

Beltrán-Orenes, Pilar; Martínez-Pastor, Esther (2016). “Grado de cumplimiento de las Leyes de transparencia, acceso y buen gobierno, y de reutilización de los datos de contratación de la Administración central española". El profesional de la información, v. 25, n. 4, pp. 557-567.

https://doi.org/10.3145/epi.2016.jul.05

Burgui-Jurío, Teresa (2010). “Qué oportunidades ofrece la 'nueva cultura digital' a las ONGD en su condición de entidades educadoras". En: Burgui-Jurío, Teresa; Erro-Sala, Javier (eds.) Comunicando para la solidaridad y la cooperación: cómo salir de la encrucijada. Pamplona: Foro Comunicación, Educación y Ciudadanía, pp. 179-200. ISBN: 978 8461439317

http://www.iudesp.uji.es/wp-content/uploads/2012/04/ teresa-burgui-jurio.pdf

Darnton, Andrew; Kirk, Martin (2011). Finding frames: New ways to engage the UK public in global poverty. London: Oxfam.

http://bit.ly/2iRADzC

Gálvez-Rodríguez, María-del-Mar; Caba-Pérez, Carmen; López-Godoy, Manuel (2016). "NGOs efficiency and transparency policy: the Colombian case". Innovar, v. 26, n. 60, pp. 67-82.

https://doi.org/10.15446/innovar.v26n60.55534

García-López, Marcial (2012). “Repensar la comunicación para la paz y la solidaridad desde lo participativo. Claves y propuestas". Razón y palabra, n. 81. 
http://bit.ly/2iRy5Bp

Herranz-de-la-Casa, José-María; Cabezuelo-Lorenzo, Francisco (2009). "Comunicación y transparencia en las organizaciones sociales. Los blogs como generadores de transparencia en las organizaciones no gubernamentales (ONGs)". Revista Icono14, n. 13, pp. 172-194.

http://www.icono14.net/ojs/index.php/icono14/article/ view/322

Karkin, Naci (2013). "Web 2.0 para la participación pública a través de los sitios de internet del gobierno". Gestión y política pública, v. 22, pp. 307-331.

http://bit.ly/2iRqyTh

España (2013). "Ley 19/2013, de 9 de diciembre, de transparencia, acceso a la información pública y buen gobierno". $B O E$, n. 295, 10 de diciembre.

https://www.boe.es/boe/dias/2013/12/10/pdfs/BOE-A-2013-12887. pdf

Manfredi-Sánchez, Juan-Luis (2016). “A major disappointment: The quest for transparency among Spanish municipalities online". International journal of media \& cultural politics, v. 12, n. 2, pp. 265-269.

https://goo.gl/HTOJOR

https://doi.org/10.1386/macp.12.2.265_7

Marí-Sáez, Víctor Manuel (2016). Comunicaciones interrumpidas. Madrid: PPC Editorial. ISBN: 9788428829922

Martínez-Osés, Pablo J.; Stronzake, Janaina; Sogge, David; Alcalde, Ana R.; Martínez-Martínez, Ignacio; Laybourn, Christina (coords.) (2011). Renovando el papel de las ONGD. Hacia la transformación social. Madrid: Plataforma 2015 y más.

http://www.2015ymas.org/IMG/pdf/Renovando_el_papel. $p d f$

Martínez-Rolán, Xabier; Piñeiro-Otero, Teresa; BaamondeSilva, Xosé-Manuel (2016). "El desafío de la transparencia en la comunicación digital. Un estudio de caso sobre las webs municipales de Galicia y Norte de Portugal". Observatorio (OBS*), v. 10 n. 4, pp. 35-55.

http://obs.obercom.pt/index.php/obs/article/view/909

Mauri-Ríos, Marcel; Ramón-Vegas, Xavier (2015). “Nuevos sistemas de rendición de cuentas de la información periodística. Exploración del escenario online español". El profesional de la información, v. 24, n. 4, pp. 380-389.

https://doi.org/10.3145/epi.2015.jul.04

Molina-Rodríguez-Navas, Pedro; Corcoy-Rius, Marta; Si-
melio-Solà, Núria (2015). "Evaluación de transparencia y calidad de la información de las entidades no lucrativas". En: Molina-Rodríguez-Navas, Pedro. Transparencia de la comunicación pública local. El mapa Infoparticipa (www.mapainfoparticipa.com). La Laguna: Sociedad Latina de Comunicación Social, pp. 113-132. ISBN 9788415968951 https://doi.org/10.4185/cac78

Molina-Rodríguez-Navas, Pedro; Lavado-Campàs, Alexandre (2015). "Indicadores de transparencia y calidad de la información de las entidades no lucrativas". En: Congreso intl cultura digital, comunicación y sociedad: Perspectivas del siglo XXI (Universidad San Jorge, Zaragoza, 16-17 marzo). http://bit.ly/2kjxtIU

Moreno-Sardà, Amparo; Molina-Rodríguez-Navas, Pedro; Corcoy-Rius, Marta; Aguilar-Pérez, Antonio; Borràs-Farran, Miquel (2013). “Infoparticip@: periodismo para la participación ciudadana en el control democrático. Criterios, metodologías y herramientas". Estudios sobre el mensaje periodístico, v. 19, n. 2, pp. 783-803.

https://doi.org/10.5209/rev_ESMP.2013.v19.n2.43471

Narberhaus, Michael (coord.) (2011). Smart CSOs: estrategias para la gran transición. Cinco puntos de apoyo para las organizaciones de la sociedad civil. Madrid: Ecodes.

http://ecodes.org/component/option,com_phocadownload/ Itemid,2/download,214/id,118/view,category

Navajo-Gómez, Pablo (2009). Planificación estratégica en organizaciones no lucrativas. Guía participativa basada en valores. Madrid: Narcea Ediciones. ISBN: 9788427715998

Plataforma de ONG de Acción Social (2013). II Plan estratégico del tercer sector de acción social, 2013-2016. Madrid: Plataforma de ONG de Acción Social.

http://www.plataformaong.org/planestrategico/ descargaRecursos. php?id=199

Santolino-Prieto, Montse (2010). "Recuperando la esencia: las ONGD como agentes de comunicación para el cambio social”. En: Burgui-Jurío, Teresa; Erro-Sala, Javier (eds.). Comunicando para la solidaridad y la cooperación: cómo salir de la encrucijada. Pamplona: Foro Comunicación, Educación y Ciudadanía, pp. 179-200. ISBN: 9788461439317 http://www.iudesp.uji.es/wp-content/uploads/2012/04/ montse_santolino.pdf

Soria-Ibáñez, María-del-Mar (2011). La comunicación de las ONG españolas: influencia de internet en el modelo estratégico de relaciones con los públicos. Tesis doctoral. Málaga: Servicio de Publicaciones Universidad de Málaga.

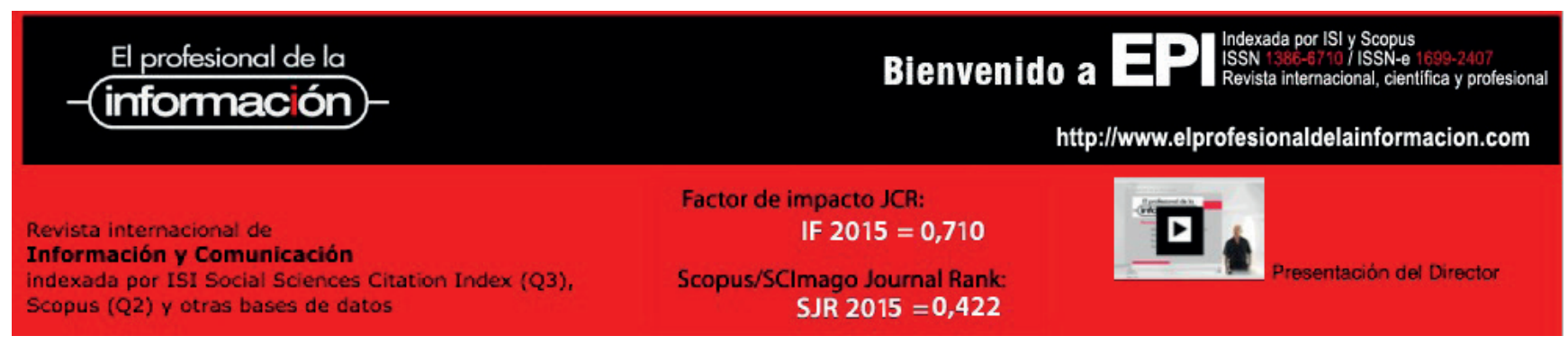

